OPEN ACCESS

Edited by:

Anders Fasth,

University of Gothenburg, Sweden

Reviewed by:

Vassilios Lougaris,

University of Brescia, Italy

Hassan Abolhassani,

Karolinska University

Hospital, Sweden

*Correspondence:

Faranaz Atschekzei

Atschekzei.Faranaz@mh-hannover.de

Specialty section:

This article was submitted to

Primary Immunodeficiencies,

a section of the journal

Frontiers in Immunology

Received: 30 August 2021 Accepted: 02 December 2021 Published: 24 December 2021

Citation:

Anim M, Sogkas G, Schmidt G, Dubrowinskaja N, Witte T, Schmidt RE and Atschekzei F (2021) Vulnerability to Meningococcal

Disease in Immunodeficiency Due to a Novel Pathogenic Missense Variant in NFKB1.

Front. Immunol. 12:767188. doi: 10.3389/fimmu.2021.767188

\section{Vulnerability to Meningococcal Disease in Immunodeficiency Due to a Novel Pathogenic Missense Variant in NFKB1}

\author{
Manfred Anim ${ }^{1,2}$, Georgios Sogkas ${ }^{1,3}$, Gunnar Schmidt ${ }^{4}$, Natalia Dubrowinskaja ${ }^{1}$, \\ Torsten Witte ${ }^{1}$, Reinhold Ernst Schmidt ${ }^{1,3}$ and Faranaz Atschekzei ${ }^{1,3^{\star}}$ \\ ${ }^{1}$ Department of Rheumatology and Immunology, Hannover Medical School, Hannover, Germany, ${ }^{2}$ Hannover Biomedical \\ Research School (HBRS), Hannover Medical School, Hanover, Germany, ${ }^{3}$ RESIST - Cluster of Excellence 2155 to Hanover \\ Medical School, Satellite Center Freiburg, Hanover, Germany, ${ }^{4}$ Department of Human Genetics, Hannover Medical School, \\ Hannover, Germany
}

$\mathrm{NF}-\mathrm{kB} 1$ deficiency is suggested to be the most common cause of common variable immunodeficiency (CVID). NFKB1 encodes for the p105 precursor protein of NF-kB1, which is converted into the active transcriptional subunit p50 through proteasomal processing of its C-terminal half upon stimulation and is implicated in the canonical NFkB pathway. Rare monoallelic NFKB1 variants have been shown to cause (haplo) insufficiency. Our report describes a novel NFKB1 missense variant (c.691C>T, p.R230C; allele frequency 0.00004953) in a family vulnerable to meningitis, sepsis, and late-onset hypogammaglobulinemia. We investigated the pathogenic relevance of this variant by lymphocyte stimulation, immunophenotyping, overexpression study and immunoblotting. The ectopic expression of p50 for c.691 C>T restricted transcriptionally active p50 in the cytoplasm, and immunoblotting revealed reduced p105/50 expression. This study shows that the deleterious missense variant in NFKB1 adversely affects the transcriptional and translational activity of NFKB1, impairing its function. Patients immunological parameters show a progressive course of hypogammaglobulinemia, which may partially account for the incomplete disease penetrance and suggest the need for closer immunological monitoring of those mutation carriers.

Keywords: common variable immune deficiency (CVID), NFKB1, Nfkb1 (p50), hypogammaglobulinemia, primary antibody deficiency (PAD)

\section{INTRODUCTION}

Primary antibody deficiencies, particularly common variable immunodeficiency (CVID), is the most common symptomatic primary immunodeficiency disorder. Patients with CVID have a highly variable clinical presentation. Besides an increased susceptibility to upper and/or lower respiratory infections, patients also present a high incidence of severe bacterial infections such as sepsis and meningitis and immune dysregulation features including lymphoproliferative, gastrointestinal and autoimmune manifestations (1-3). Before diagnosing CVID, secondary cause of antibody deficiency 
(SAD) must be excluded in clinical practice (4). So far, several monogenic defects such as ICOS, CD19, CD20, CD21, CD27, CD81, IL21, IL21R, LRBA, PRKCD, RAC2, TNFSF12, CTLA4, PLCG2, NFKB1, NFKB2, PIK3CD, PIK3R1, VAV1, BLK, IKZF1, IRF2BP2, as well as mutations in TNFRSF13B and TNFRSF13C have been identified in CVID $(1,5,6)$. Recently NF- $\kappa B 1$ haplo (insufficiency) has been described as a novel monogenic cause of CVID (6). Several independent studies have reported that lossof-function variants in NFKB1 are probably the common cause of antibody deficiency with a highly variable clinical and immunological presentation (7). The nuclear factor of kappa light polypeptide gene enhancer in B cells (NF- $\kappa$ B) is a family of closely related ubiquitous transcription factors that regulate an extensive array of genes involved in different immune and inflammatory responses. This family is composed of five structurally related proteins including NF- $\kappa B 1$ (p50/p105), NFКB2 (p52/p100), RelA (p65), c-Rel, and RelB that mediates transcription of target genes by binding to a specific DNA element, $\kappa \mathrm{B}$ enhancer, as various hetero- or homo-dimers (8). The NF- $\kappa \mathrm{B}$ proteins are usually sequestered in the cytoplasm by a family of inhibitory proteins, including I $\mathrm{I} B$ family members and related proteins defined by the presence of ankyrin repeats. Two different signal pathways have been proposed for NF- $\mathrm{KB}$ activities, the classical/canonical or non-canonical pathway. Upon stimulation of classical pathway by Toll-like-receptors, or B and T cell receptors, the IKKB subunit phosphorylates and polyubiquitinates, leading to its degradation by the $26 \mathrm{~S}$ proteasome (8) and translocation of p50 into the nucleus to exert its function as a transcription factor (9).

We have recently identified the pathogenic impact of identified NFKB1 deleterious variants in our PAD cohort; we have shown that missense mutation in the NFKB1 causes late-onset PAD by impairing the function of the transcriptionally active p50 (10). We identified further a previously uncharacterized NFKB1 missense variant in a family with a history of meningococcal meningitis and late-onset hypogammaglobulinemia by next-generation sequencing (NGS) as the only predicted deleterious variant within genes of inborn errors of immunity. Hence in the current work, we mainly focused on the relevance of this NFKB1 missense variant (c.691 C>T, p.R230C) by immunophenotyping, immunoblotting, and ectopic expression assays.

\section{MATERIALS AND METHOD}

\section{Ethical Aapproval}

The institutional medical ethical committee at Hannover Medical School approved the study (ethics approval number: Nr.8875_BO_K_2020). The written consent of all study participants was obtained.

\section{Isolation of Genomic DNA and Sequencing Methods}

Genomic DNA (gDNA) was isolated from peripheral blood of patients and healthy donors with QIAamp Kit (QIAamp DNA Blood Midi Kit; Lot\# 16902455; Qiagen). Whole exome sequencing (WES) was performed on genomic DNA samples from patients S1 and S2 as described previously (11). Briefly, the concentration and quality of the purified genomic DNA (gDNA) was determined with an Agilent Technologies 2100 Bioanalyzer (Agilent Technologies, Santa Clara, CA, USA). The DNA sequencing library consisted of $100 \mathrm{ng}$ fragmented gDNA and was generated with Agilent SureSelectXT Reagent Kits v5 UTR (70 $\mathrm{Mb}$ ) according to the manufacturer's protocols (Illumina, San Diego, CA, USA). Libraries were sequenced on an Illumina HiSeq2500 platform using TruSeq SBS Kit v3-HS (200 cycles, paired end run) with an average of $12.5 \times 106$ reads per single exome (mean coverage: 50X). The GATK-Pipeline (GenomeAnalysisTK-1.7) was applied for read quality trimming, read alignment to reference (GRCh37/hg19) and quality trimmed variant calling. Variant annotation was performed using Gsvar software. We selected for rare variants with low minor allele frequency $(\mathrm{MAF}<0.05)$. Sanger sequencing was performed by (Eurofins Genomics) to validate the identified rare NFKB1 variant and its co-segregation with disease phenotype in this family using the primers: forward; 5'-GTCTATTCTTGGTGTGCCCC-3' and reverse; 3'TGCAGCAGACCAAGGAGATG-5'.

\section{PBMCs Isolation}

Whole blood was collected from both patients and healthy control. PBMCs were isolated using the standard centrifugation method. Briefly, whole blood was mixed with PBS in a 1:2 ratio, and the diluted cell suspension was gently layered on the Ficol-plaque separation gradient. Centrifugation was carried out at $1000 \mathrm{xg}$ for 20 mins with no break at $21^{\circ} \mathrm{C}$. The mononuclear cell layer was carefully removed, transferred into new $50 \mathrm{ml}$ falcon tubes, and washed with PBS. Cells were either stored in 10\% DMSO or immediately used.

\section{Lymphocyte Stimulation Assays}

For stimulation experiments, PBMCs were treated with phorbol 12 -myristate 13 -acetate (PMA; $50 \mathrm{ng} / \mathrm{ml})$ and ionomycin $(1 \mu \mathrm{g} /$ $\mathrm{ml}$ ) and incubated for $30 \mathrm{~min}$ at $37^{\circ} \mathrm{C}$ and $5 \%$ carbon dioxide. After incubation cells were harvested and used immediately for total protein extraction.

For standard $\mathrm{T}$ cell proliferation assays, PBMCs were stimulated with phytohemagglutinin (PHA), concanavalin A (ConA), pokeweed mitogen (PWM), purified protein derivative (PPD), interleukin 2 (IL-2) and anti-CD3mAb as described previously $(10,12)$.

\section{Cell Culture and Transfection}

Human Embryonic Kidney 293 (HEK 293) cells were cultured in Dulbecco's modified Eagle's medium (DMEM) supplemented with $10 \%$ heat-inactivated fetal bovine serum (FBS), $1 \%$ penicillin/streptomycin, and $1 \%$ Sodium pyruvate (Invitrogen) at $37^{\circ} \mathrm{C}$ with $5 \% \mathrm{CO} 2$. A day before transfection, $5 \times 10^{\wedge} 5 \mathrm{HEK}$ 293 cells/ well were seeded into a six-well plate and cultured at $37^{\circ} \mathrm{C}$. Transfection was carried out using a $2 \mu \mathrm{g}$ plasmid, each using $\mathrm{x}$-tremegene hp DNA transfection reagent (Merck, 6366244001) following the manufacturer's protocol utilizing eGFP plasmid expression vector and Opti-Mem as a negative 
control. Transfected cells were selected two days posttransfection using $400 \mu \mathrm{g} / \mathrm{m}$ G418 sulfate.

\section{Cloning}

Plasmids encoding either p105 or p50 wild type and mutant with N-terminal GFP tag were cloned into pc.DNA3.1 (+)-N-eGFP (Genescript). Competent E. coli (NEB, 5-alpha) were transformed with the vectors constructs, and plasmids were isolated with QIAprep Spin Mini Kit (Qiagen).

\section{Fluorescence Staining and Confocal Imaging}

HEK293 cells that were transiently expressing eGFP alone or $\mathrm{N}$ terminally EGFP-tag p50-wt or p50-R230C mutant were fixed with $4 \%$ paraformaldehyde for 20 mins at RT followed by $3 \mathrm{X}$ washing with PBS and permeabilized with $0.1 \%$ Triton X-100. The nuclei were stained with DAPI. For microscopy analysis, glass coverslips were mounted with a drop of the fluorescencemounting medium (DAKO). Confocal fluorescence images were taken on an Olympus FV1000 laser-scanning microscope. Images were evaluated and processed with Fiji software.

\section{Western Blotting and qRT-PCR}

Total RNA and proteins were isolated from transfected HEK293 cells or subjects' and healthy control PBMCs using NE-PER Nuclear and cytoplasmic extraction reagent (Thermo Scientific; lot \# TA259812) and Allprep DNA/RNA micro-kit (Qiagen; \#80284), respectively. Proteins were separated on $7.5 \%$ MiniProtean TGX (BIO-RAD; \#4561024) and transferred onto Invitrogen PVDF membranes (Thermo Scientific). Both p105 and p50 proteins were detected using a primary polyclonal rabbit antibody directed against the $\mathrm{N}$-terminal amino acids (Cell Signaling; \#3035). A monoclonal rabbit antibody was used to detect levels of phosphorylated p105 at serine 933 (\#4806; Cell Signaling). Horseradish-peroxidase-coupled goat anti-rabbit secondary antibody (\#6721; Abcam) was used to detect signals via enhanced chemiluminescence (SuperSignal West Dura Extended Duration Substrate; Thermo Fischer). For the loading controls rabbit beta actin (\#12620; cell signaling) and histone H3 (\#12648; cell signaling) directly coupled to horseradish-peroxidase was used. Rabbit antibody against beta-actin directly coupled to horseradish peroxidase was used as a loading control. For quantitative Real-Time polymerase chine reaction (qRT-PCR), 1 $\mu \mathrm{g}$ of total RNA was used for cDNA synthesis. Reverse transcription was performed using High-Capacity cDNA Reverse Transcription Kit (Applied biosynthesis, Lithuania) following the manufacturer's protocol. No RNA and no reverse transcriptase served as the negative control. Expression levels of NFKB1 and GAPDH transcript were quantified using Taqman Gene Expression Master Mix with custom-made probes were used for the qRT-RT assay. The average changed in threshold cycle values was determined for each sample relative to the endogenous GAPDH levels and compared with the control.

\section{Statistics}

Statistical analyses were performed using Graph Pad Prism, version 8. One-way analysis of variance (ANOVA) test was used to compare $N F \kappa B 1$ expression. Densitometry analyses of western blots were performed with ImageJ software (Version $1.52 \mathrm{v}$ ), and the graphs were prepared using Microsoft Excel 365.

\section{RESULTS}

\section{Identification of NFKB1 Missense Variants by tNGS}

We identified a NFKB1 deleterious missense variant (691 C>T, p.R230C) in a family with antibody deficiency (Figure 1A). This variant was confirmed by Sanger sequencing (Figure 1B) and predicted to be deleterious by in silico tools.

\section{Clinical History}

A 23-year-old patient (S2) born to non-consanguineous Caucasian healthy parents of German descent presented to our Immunology outpatient clinic due to a history of meningococcal meningitis with sepsis (Waterhouse-Friderichsen syndrome) at the age of 10 years. He additionally had a parechovirus meningitis at the age of 20 years. Except for the aforementioned two meningitides and the identification of 2-3 isolated boils through the physical examination, his infection record was inconspicuous. This patient's two years younger brother (S3) also suffered from meningococcal meningitis with sepsis at the age of 17 years, at a different time point than his brother. He additionally reported recurrent bronchitis and one pneumonia. On the bases of immunological investigations S2 were consistent with the diagnosis of a combined immunodeficiency (CID), whereas S3 was diagnosed with common variable immunodeficiency (CVID). Findings of immunological investigations of both brothers are summarized in Table 1. Patients' clinical and immunological values are regularly monitored and examined in our clinic. Furthermore, none in the family had a history of severe or recurrent infections.

\section{Missense Variant $691 \mathrm{C}>\mathrm{T}$, p.R230C Causes p105/p50 Reduction and Impair p50 Nuclear Translocation}

Both the two brothers (S2/3) were identified to carry the missense variant $691 \mathrm{C}>\mathrm{T}$, p.R230C in NFKB1 located on the $\mathrm{N}$-terminal of the RHD (Figure 1C). This variant refers to the group of NFKB1 mutations, affecting both the precursor p105 and the mature p50 as we described previously (10). HEK293 cells were transiently transfected with EGFP-fused mutant proteins or EGFP-fused wild-type proteins. Immunoblotting and qRT-PCR were carried out. A reduced mRNA level and a drastically reduced protein level (approximately 92\%) were observed for p105/50 (Figures 2B; Supplementary Figure 1) in transfected HEK293 cells. In transfected HEK293 cells, the EGFP-fused p50-R230C mutant showed an aberrant localization into the nucleus while the wildtype localization was normal (Figure 2A). More importantly, the expression of p50 using the EGFP-fused p50-R230C mutant in HEK293 showed that p50 expression in the mutant was reduced compared to the wild type (Figure 1C). These results indicate that the functional defect influences both the precursor p105 and mature p50. 
A

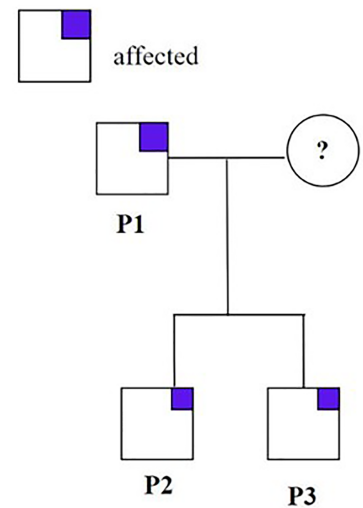

B

Subject

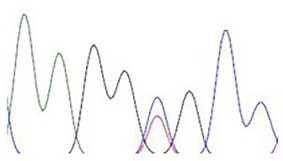

Control

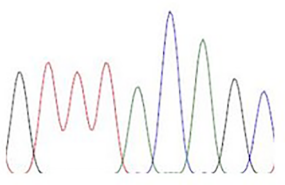

C
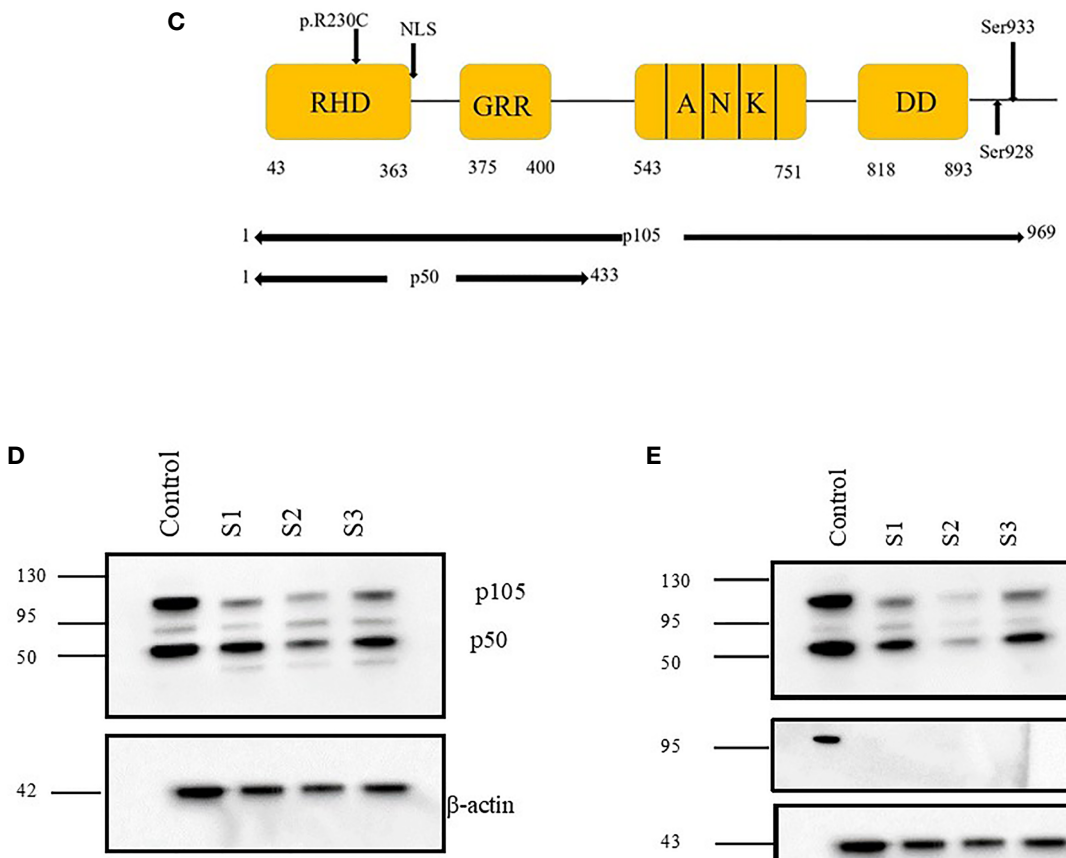

E

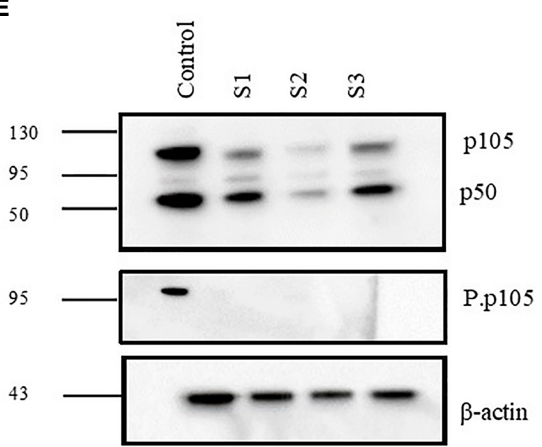

FIGURE 1 | Monoallelic NFKB1 missense mutation in a family with late-onset antibody deficiency. (A) Segregation of NFKB1variant was analyzed by sequencing genomic PCR product and revealed an autosomal-dominant inheritance in families with reduced clinical penetrance. The analysis excluded the mother of patients because of material lack. (B) Sanger sequencing of genomic PCR products results in the chromatogram of missense variant and wild type (WT) (C) Structure of NFKB protein showing the position of the identified mutation. (D) Immunoblotting was performed in PBMCs of subjects (S1, S2, and S3) and healthy control (HC), and the expression of p105/50 was evaluated. The expression of p105 was reduced for all the subjects compared to the control. However, the expression of p50 was reduced in S2. (E) PBMCs from HC and S1, S2 and S3 were stimulated with PMA; $50 \mathrm{ng} / \mathrm{ml}$ and ionomycin; $1 \mu \mathrm{g} / \mathrm{ml}$ and the expression of p105/50 evaluated. There were no significant changes in the p105/50 expression after stimulation in the subjects; however, p105 phosphorylation at serine 933 was detected in only the $\mathrm{HC}$ but not in the subject. Beta-actin was used as a cytoplasmic loading control.

PBMCs from mutation carriers $(\mathrm{n}=3)$, healthy control $(\mathrm{n}=1)$ were stimulated with PMA plus ionomycin and protein were extracted from cytoplasm and nucleus and analyzed by western blotting. Representative results are shown (Figures 1D, E). p105 amounts, phosphorylation of $\mathrm{p} 105$, and processing to $\mathrm{p} 50$ are severely reduced compared to control. The $\beta$-actin control confirms equal loading.

\section{DISCUSSION}

\section{The Pathogenic NFKB1 c.691 C>T, p.R230C Variant Causes Progressive Late-Onset Antibody Deficiency}

This study assayed the pathogenicity of a deleterious missense NFKB1 variant, c.691 C>T, detected in two brothers (S2/3) and 
TABLE 1 | Clinical and immunological characteristics of studied subjects with NF-кB1 LOF variant.

\begin{tabular}{|c|c|c|c|c|}
\hline & S.1 & S.2 & S.3 & $\begin{array}{c}\text { Reference } \\
\text { range }\end{array}$ \\
\hline Year of birth & 1974 & 1998 & 2000 & \\
\hline Sex & male & male & male & \\
\hline Infections & $\begin{array}{l}\text { None } \\
\text { reported }\end{array}$ & $\begin{array}{l}\text { Neisseria meningitidis meningitis and sepsis (Waterhouse- } \\
\text { Friderichsen-syndrome); Parechovirus meningitis; isolated } \\
\text { furuncle }\end{array}$ & $\begin{array}{l}\text { Neisseria meningitidis sepsis (Waterhouse- } \\
\text { Friderichsen-syndrome); one pneumonia; twice } \\
\text { bronchitis within a year }\end{array}$ & \\
\hline \multicolumn{5}{|c|}{ 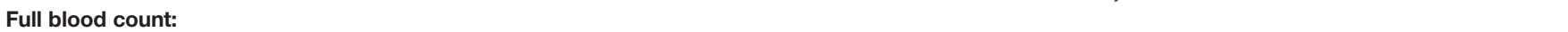 } \\
\hline WBC (cells/ul) & 6100 & 8000 & 5700 & $\begin{array}{l}4800- \\
12000\end{array}$ \\
\hline Lymphocytes (cells/ $\mu$ l) & 1200 & 1160 & 940 & $1100-4500$ \\
\hline Lymphocytes (\% WBC) & 16.0 & 14.8 & 16.5 & $20-44$ \\
\hline Monocytes (\% WBC) & 8.7 & 8.8 & 8.3 & $2-9.5$ \\
\hline Neutrophils (\% WBC) & 72.9 & 74 & 72.2 & $42-77$ \\
\hline \multicolumn{5}{|c|}{ Phenotypic profile of peripheral blood lymphocytes: } \\
\hline $\begin{array}{l}\text { CD3+ T cells }(\% \\
\text { lymphocytes) }\end{array}$ & 36.6 & 50 & 69 & $55-83$ \\
\hline $\begin{array}{l}\text { CD4+ T cells (\% } \\
\text { lymphocytes) }\end{array}$ & 45 & 31.6 & 45.4 & $55-83$ \\
\hline $\begin{array}{l}\text { CD8+ T cells ( } \% \\
\text { lymphocytes) }\end{array}$ & 29.3 & 15.1 & 18.6 & $10-39$ \\
\hline $\begin{array}{l}\text { CD19+ B cells ( } \% \\
\text { lymphocytes) }\end{array}$ & 10.7 & 12 & 5 & $6-19$ \\
\hline$\gamma \delta$ T-cell (\% T cells) & 27.7 & 15.1 & 9.6 & $<10 \%$ \\
\hline $\begin{array}{l}\text { CD3+CD56+ NK cells (\% } \\
\text { lymphocytes) }\end{array}$ & 6.9 & 22.3 & 22.3 & $7-31$ \\
\hline \multicolumn{5}{|c|}{ Phenotypic profile of peripheral blood CD4+T cells: } \\
\hline $\begin{array}{l}\text { naive CD4+ T cells ( } \% \text { of } \\
\text { CD4+ T cells) }\end{array}$ & 46.9 & 74.6 & 67.8 & $(49-72)$ \\
\hline $\begin{array}{l}\text { memory CD4+ T cells (\% } \\
\text { CD4+ T cells) }\end{array}$ & 46.5 & 17.4 & 14.3 & $34-71$ \\
\hline $\begin{array}{l}\text { recent thymic emigrant } \\
\text { (RTE) T helper cells }(\% \\
\text { CD4+ T cells) }\end{array}$ & 20.6 & 48.4 & 55 & $42-64$ \\
\hline \multicolumn{5}{|c|}{ Phenotypic profile of peripheral blood CD8+T cells: } \\
\hline $\begin{array}{l}\text { early effector memory CD8+ } \\
T \text { cells ( } \% \text { of CD8+ T cells) }\end{array}$ & 13.9 & 5.8 & 11.5 & $2.9-16$ \\
\hline $\begin{array}{l}\text { late effector memory CD8+ } \\
\text { T cells ( } \% \text { CD } 8+T \text { cells) }\end{array}$ & 9.5 & 1.6 & 4.8 & $2.6-58$ \\
\hline \multicolumn{5}{|c|}{ Phenotypic profile of peripheral blood CD19+ B cells: } \\
\hline Naive B cells ( $\%$ B cells) & 78.9 & 71.1 & 70.7 & $29-93$ \\
\hline $\begin{array}{l}\text { lgM+ memory B cells (\% B } \\
\text { cells) }\end{array}$ & 5.8 & 5.9 & 7.1 & $2-25$ \\
\hline $\begin{array}{l}\text { class-switched B cells (\% B } \\
\text { cells) }\end{array}$ & 6.9 & 14.6 & 14.3 & $3-23$ \\
\hline $\begin{array}{l}\text { transitional B cells (\% B } \\
\text { cells) }\end{array}$ & 3.7 & 1.5 & 1.2 & $0.6-4-6$ \\
\hline plasmablasts (\% B cells) & 5.2 & 7.6 & 5.5 & $0.4-3.6$ \\
\hline $\mathrm{CD} 21^{\text {low }} \mathrm{B}$ cells (\% B cells) & 5.2 & 3.7 & 2.1 & $1-26$ \\
\hline \multicolumn{5}{|l|}{ Immunoglobulins: } \\
\hline $\lg G(g / \operatorname{l})$ & 6.2 & 9.64 & 6.95 & $7-16$ \\
\hline $\lg A(g / l)$ & 0.51 & 0.66 & 0.53 & $0.7-4$ \\
\hline $\operatorname{lgM}(g / l)$ & 0.5 & 0.91 & 0.62 & $0.4-2.3$ \\
\hline $\begin{array}{l}\text { Pneumococcal antibody } \\
(\mathrm{mg} / \mathrm{l})^{\star \star}\end{array}$ & n.m. & 110 & 251.7 & $39.4-100.5$ \\
\hline Tetanus antibody $(\mathrm{IU} / \mathrm{ml})^{\star \star}$ & n.m. & 1.2 & 0.8 & $>1.1$ \\
\hline \multicolumn{5}{|l|}{ Granulocytes function test: } \\
\hline $\mathrm{C} 3 \mathrm{c}(\mathrm{g} / \mathrm{l})$ & n.m. & 1.01 & 1.4 & $0.9-1.8$ \\
\hline $\mathrm{C} 4(\mathrm{~g} / \mathrm{l})$ & n.m. & 0.18 & 0.14 & $0.1-0.4$ \\
\hline CH50 (U/ml) & n.m. & $>60$ & $>60$ & $31.6-57.6$ \\
\hline AP50 (\%) & n.m. & 110 & 92 & 60-102 \\
\hline
\end{tabular}

n.m, not measured.

**values after booster vaccination.

Values in bold deviate from reference range. 
A

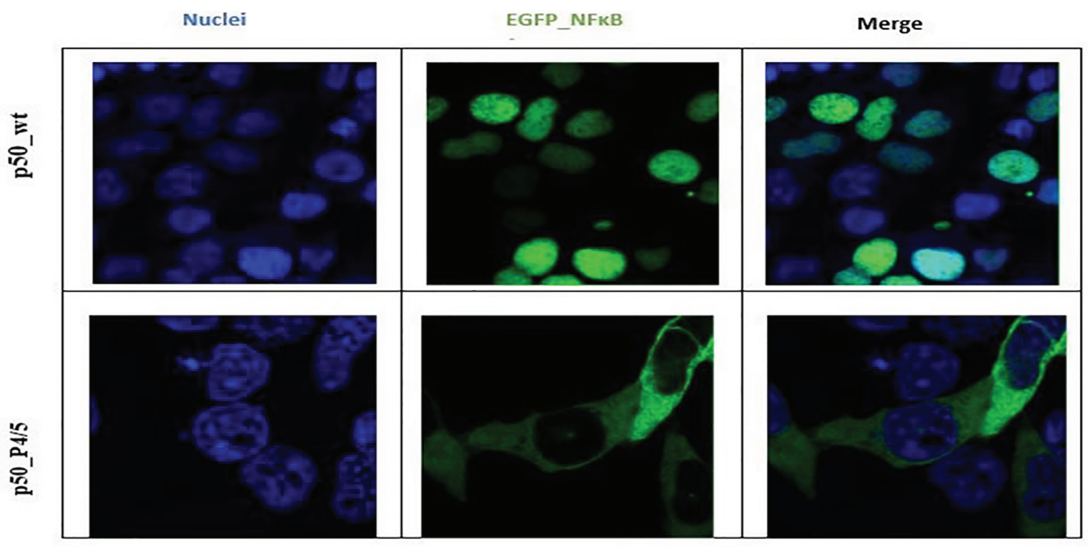

B
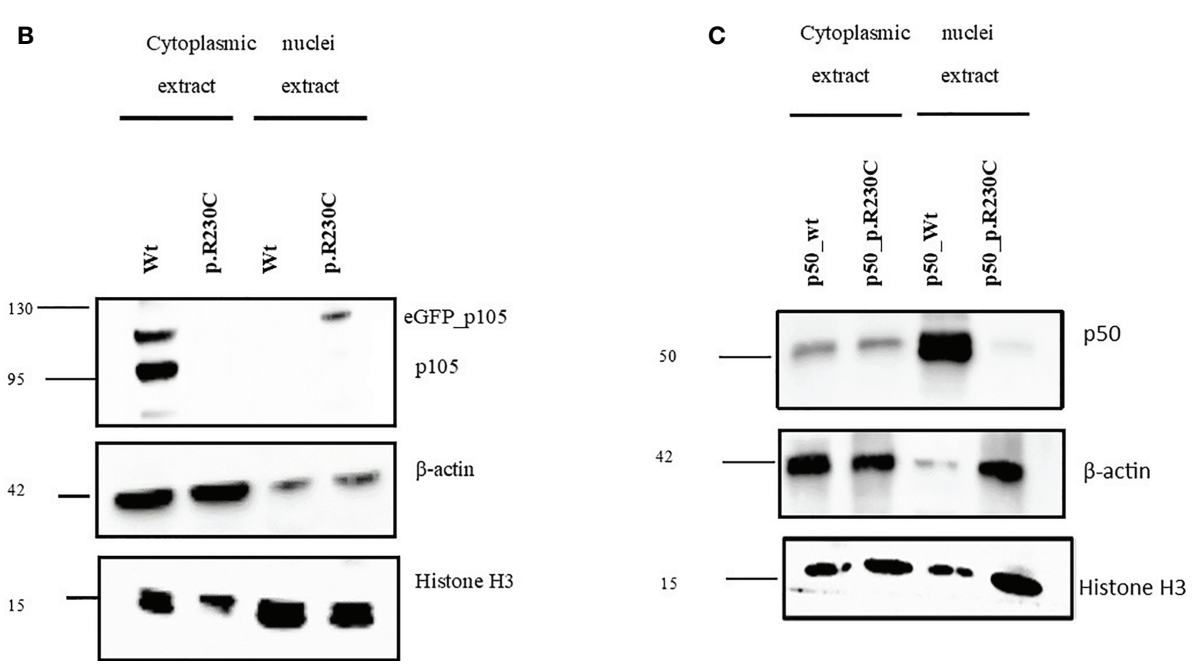

FIGURE 2 | NFkB1 expression in and nuclear translocation in transfected HEK293 cells. Total proteins were extracted from transfected HEK293 cells to determine the expression of p105/50. (A) For microscopic analysis of NFkB1-p50 translocation into the nucleus, HEK293 cells transiently expressing the eGFP-p50 wild type and mutant were used. Nuclei were stained with DAPI. The wild-type p50-construct localizes into the nucleus while the mutant p50-construct was retained in the cytoplasm. (B) Western blot to analyze the effect of the variant on the expression of p105/50 in the transfected HEK293 cells. The expression in the wild type was prominent in the wild type, while no noticeable expression in the variant was observed. (C) p50 expression in HEK293 showed an increase in wild-type and a decrease in the mutant (p. R230C) in the nuclear extract.

their father (S1) from a German family with variable clinical presentations but a shared history of meningococcal meningitis with sepsis and viral meningitis in the different points of their childhood and adolescence. Later, both developed CID and CVID, respectively. The variant is located in the $\mathrm{N}$-terminal part of p105; using the in vitro transfection model, we found an impaired nuclear translocation of active p50.

Neisseria meningitidis is a restricted human bacterium and a common nasopharynx colonizer. The bacterium is often harmless but, in rare cases, can cause life-threatening meningitis and sepsis. There is solid evidence for the role of host genetics in predisposition to meningococcal infection. So far, monogenic defects in the terminal component of complement and polymorphism in the $\mathrm{CFH} / \mathrm{CFHR} 3$ region have been described to be associated with susceptibility to meningococcal meningitis (13). An association between meningococcal meningitis and NFKB1 is not reported yet.
Recently performing the ectopic expression in HEK293T cells, we have shown that frameshift mutations in $\mathrm{N}$ terminal "p50 half" of p105 lead to seriously truncated proteins that lack the nuclear localization sequence (NLS) and consequently cause rapid proteasomal degradation (10). The overall expression levels of both p105 and p50 were decreased by about 50\% compared to healthy controls, as previously reported for several NFKB1 haploinsufficiency variants $(6,7,10)$. Almost all patients with alteration in NFKB1 causing p50 haploinsufficiency presented with diverse clinical manifestations, mainly autoimmunity, lymphoproliferative disorders, splenomegaly, CMV infections, and malignancies besides antibody deficiency (14). Our reported patient with truncating mutation presented the most severe phenotype; his sons carry the same mutation; however, one displayed mild IgG while the other showed autoimmune thyroiditis and asthma bronchial (10). Therefore, both are 
under closer follow-up because IgG deficiencies can develop into CVID over time, and the age-dependent exhibition of the NF$\kappa \mathrm{B} 1$-related phenotype has been reported (14).

Deleterious mutations in the $\mathrm{N}$ terminal part of $\mathrm{p} 105$, including frameshift and truncations, have been shown to cause (haplo) insufficiency, whereas examining the pathogenic relevance of a vast number of NFKB1 missense variants in patients with immunodeficiency remained scarce. The recorded variant p.H67R reduced nuclear entry of p50 and showed decreased transcriptional activity in a luciferase reporter assay (15). The three rare substitutions (p.I281M; p.V98D, p.I87S) identified in sporadic CVID have been shown to reduce p50 levels and affect protein stability. We have recently demonstrated that the rare p.R157P variant reduced p105/p50 expression in the patient's derived cells, whereas the EGFP-fused mutant p50 revealed an aberrant intranuclear pattern (10). Most recently, the characterization of p.Y350C substitution in transfected HEK293T cells has shown decreased p105 expression, indicating an accelerated decay, although forced expression of mutant p50 affected nuclear translocation (16). Recently, Li et al. evaluated the functional impact of 365 NFKB1 variants utilizing a reporter assay and showed that deleteriousness of monoallelic variants in NFKB1 lies on haploinsufficiency. Characterized NFKB1 variants included missense LOF or hypomorphic variants, which all - similar to the variant presented in the present work were localized at the RHD domain of p105/p50 (17).

In the present study, we investigated the impact of variant c.691 C>T, p. R230C by overexpression assay of EGFP-fused $\mathrm{NF \kappa B} 1$ proteins. HEK293 cells were transiently transfected with EGFP-fused mutant proteins or EGFP-fused wild-type proteins. Confocal microscopy analysis also showed that p50 was sequestered in the cytoplasm, whereas wild-type localization was in the nucleus (Figure 2A). Immunoblotting revealed reduced expression of mutant p105 compared to the wild type (Figure 2B) and a drastic diminish of p50 mutant in the nucleus compared to the wild-type counterpart (Figure 2C), indicating the functional defect affects both and predominately the p50 localization into the nucleus.

The assessment of NFKB1 protein levels in the PBMCs of the father (S1) and the two brothers (S2/3) was investigated. We observed a reduction in $\mathrm{p} 105 / 50$ in all the missense variants carriers compared to the control (Figure 1D). More importantly, the expression of p50 was substantially reduced for S2. Although the patients' father carries the same mutations with a reduced p105 expression, he is clinically unaffected. This observation is consistent with previous studies that observed a reduction in p105/50 expression in clinically and non-clinically affected family members (18).

Indeed, the diverse clinical phenotypes are seen in these brothers harbouring the same mutation refer to the incomplete penetrance nature of the disease and might be explained by additional factors such as environmental and or epigenetic alterations (19) that necessitate further investigations. With the coming of NGS in several independent studies, NFKB1 mutations have been described as the most frequent monoallelic genetic cause of PAD with variable clinical phenotype, even within the same affected family (20). In addition, recent cohort studies on patients with NFKB1 variants have shown that infections, lymphoproliferative disorders, autoimmune diseases, and malignancies are the most common and age-dependent manifestations in these patients (14). Besides its role in regulating B-cell activities such as differentiation and proliferation, NF-kB1 is also crucial for T-cell activation, antigen presentation, and regulation of tissue-specific autoimmunity (21). Thus reduction in p105/p50 expression may play a role in autoimmunity, lymphadenopathy, and splenomegaly as observed in mice lacking p105 expression (22). Our patients currently present milder clinical phenotypes with hypogammaglobulinemia, moderate lymphopenia, and reduced CD4+T cells. Since family members, carrying deleterious NFKB1 variants with moderate phenotype are at high risk for autoimmunity and malignancy, a closer follow-up for early initiation of IgG substitution therapy to prevent complications is strongly recommended.

\section{CONCLUSION}

Pathogenic variants within RHD impairs the p50 nuclear translocation and, therefore, might diminish the functions of p50, resulting in progressive antibody deficiency and suggests the need for closer monitoring and counselling of mutation carriers. This study is a further crucial extension of our knowledge in the NF- $\kappa$ B1-related phenotype.

\section{DATA AVAILABILITY STATEMENT}

The original contributions presented in the study are publicly available. This data can be found here: https:/www.ncbi.nlm.nih. gov/bioproject/PRJNA788443.

\section{ETHICS STATEMENT}

The studies involving human participants were reviewed and approved by the institutional medical ethical committee at Hannover Medical School approved the study (ethics approval number: Nr.8875_BO_K_2020). The written consent of all study participants was obtained. The patients/participants provided their written informed consent to participate in this study.

\section{AUTHOR CONTRIBUTIONS}

Research design, FA and RS. Sample collection, FA, GeS, and MA. WES, GuS. NGS data analysis, FA. Performance of functional experiments and data analysis, MA. Writing and contributing to writing of the manuscript FA, M.A, and all authors. All authors contributed to the article and approved the submitted version. 


\section{FUNDING}

This project was funded by the Deutsche Forschungsgemeinschaft (DFG, German Research Foundation) under Germany's Excellence Strategy-EXC 2155 'RESIST'-Project ID 39087428 and the German network for multi-organ autoimmune diseases GAIN_ 01GM1910A. GeS receives funding from the Young Academy Clinician/Scientist program of Hannover Medical School, Germany and the Rosemarie-Germscheid foundation. All authors and this project are supported by the German Center for Infection Research (DZIF TTU 07.801).

\section{ACKNOWLEDGMENTS}

We thank all nurses, physicians and documentation personnel of the outpatient clinics of the Department of Rheumatology and Immunology of the Hannover Medical School for collecting blood samples, informing the patients about the study and documenting patients' medications. We thank Sabine Buyny for her excellent technical assistance.

\section{REFERENCES}

1. Bousfiha A, Jeddane L, Al-Herz W, Ailal F, Casanova JL, Chatila T, et al. The 2015 IUIS Phenotypic Classification for Primary Immunodeficiencies. J Clin Immunol (2015) 35:727-38. doi: 10.1007/ s10875-015-0198-5

2. Baldwin AS. The NF- $\kappa b$ and I $\mathrm{b}$ Proteins: New Discoveries and Insights. Annu Rev Immunol (1996) 14. doi: 10.1146/annurev.immunol.14.1.649

3. Bogaert DJA, Dullaers M, Lambrecht BN, Vermaelen KY, De Baere E, Haerynck F. Genes Associated With Common Variable Immunodeficiency: One Diagnosis to Rule Them All? J Med Genet (2016) 53:575-90. doi: 10.1136/jmedgenet-2015-103690

4. Chinen J, Shearer WT. Secondary Immunodeficiencies, Including HIV Infection. J Allergy Clin Immunol (2010) 125:S195-203. doi: 10.1016/ j.jaci.2009.08.040

5. Schubert D, Bode C, Kenefeck R, Hou TZ, Wing JB, Kennedy A, et al. Autosomal Dominant Immune Dysregulation Syndrome in Humans With CTLA4 Mutations. Nat Med (2014) 20:1410-6. doi: 10.1038/nm.3746

6. Fliegauf M L, Bryant V, Frede N, Slade C, Woon ST, Lehnert K, et al. Haploinsufficiency of the NF-kb1 Subunit P50 in Common Variable Immunodeficiency. Am J Hum Genet (2015) 97:389-403. doi: 10.1016/ j.ajhg.2015.07.008

7. Tuijnenburg P, Lango Allen H, Burns SO, Greene D, Jansen MH, Staples E, et al. Loss-Of-Function Nuclear Factor kb Subunit 1 (NFKB1) Variants Are the Most Common Monogenic Cause of Common Variable Immunodeficiency in Europeans. J Allergy Clin Immunol (2018) 142(4):1285-96. doi: 10.1016/j.jaci. 2018.01.039

8. Vallabhapurapu S, Karin M. Regulation and Function of NF- $\kappa b$ Transcription Factors in the Immune System. Annu Rev Immunol (2009) 27:369-78. doi: 10.1146/annurev.immunol.021908.132641

9. Shih VF-S, Tsui R, Caldwell A, Hoffmann A. A Single Nfkb System for Both Canonical and Non-Canonical Signaling. Cell Res (2011) 21:86-102. doi: $10.1038 / \mathrm{cr} .2010 .161$

10. Schröder C, Sogkas G, Fliegauf M, Dörk T, Liu D, Hanitsch LG, et al. LateOnset Antibody Deficiency Due to Monoallelic Alterations in NFKB1. Front Immunol (2019) 10:2618. doi: 10.3389/fimmu.2019.02618

11. Sogkas G, Fedchenko M, Dhingra A, Jablonka A, Schmidt RE, Atschekzei F. Primary Immunodeficiency Disorder Caused by Phosphoinositide 3-Kinase $\delta$ Deficiency. J Allergy Clin Immunol (2018) 142:1650. doi: 10.1016/j.jaci. 2018.06.039

\section{SUPPLEMENTARY MATERIAL}

The Supplementary Material for this article can be found online at: https://www.frontiersin.org/articles/10.3389/fimmu.2021. 767188/full\#supplementary-material

Supplementary Figure 1 | Total RNA was extracted from transfected HEK293 cells to determine the expression of p105 at the mRNA level. (A) Quantitative-Real time PCR showed a reduction in the transcript levels of p105 in the mutant compared to the wild type. Delta delta Ct was then determined by subtracting $\Delta \mathrm{Ct}$ (variant) from $\Delta \mathrm{Ct}$ (wild type). Fold change in expression was calculated using 2- $\Delta \Delta \mathrm{Ct}$.

Supplementary Figure 2 | Densitometry analysis. Densitometry analysis was performed for p105 and p50 expression in the transfected HEK293 cells and PBMCs. (A) Expression of p105 in HEK293 cells transfected with wild type NFkB1 and mutant (p.R230C). (B) Expression of p50 in HEK293 cells transfected with wild type NFkB1 and mutant (p.R230C) showed a reduction in the p50 by immunoblotting. Both p105 and p50 were drastically reduced in the mutant compared to the wild type. (C) Expression of p105/50 in the PBMCs of healthy control $(\mathrm{HC})$ and mutation carriers (S1-S3). Densitometry analysis revealed lowered expression in all subjects compared to the control. (D) PBMCs from $\mathrm{HC}$ and S1, S2 and S3 were stimulated with PMA; $50 \mathrm{ng} / \mathrm{ml}$ and ionomycin; $1 \mu \mathrm{g} / \mathrm{ml}$ and the expression of p105/50 examined. There was no significant changes in the p105/50 expression after stimulation.

12. Kittner JM, Jacobs R, Buyny S, Peest D, Stoll M, Schmidt RE. Adult Onset of T-Cell Deficiency With Impaired CD2 Expression Complicated by Rhodococcus Infection: A Case Report. Ann Allergy Asthma Immunol (2007) 98:294-8. doi: 10.1016/S1081-1206(10)60722-3

13. Hodeib S, Herberg JA, Levin M, Sancho-Shimizu V. Human Genetics of Meningococcal Infections. Hum Genet (2020) 139:961-80. doi: 10.1007/s00439-02002128-4

14. Lorenzini T, Fliegauf M, Klammer N, Frede N, Proietti M, Bulashevska A, et al. Characterization of the Clinical and Immunologic Phenotype and Management of 157 Individuals With 56 Distinct Heterozygous NFKB1 Mutations. J Allergy Clin Immunol (2020) 146:901-11. doi: 10.1016/ j.jaci.2019.11.051

15. Kaustio M, Haapaniemi E, Göös H, Hautala T, Park G, Syrjänen J, et al. Damaging Heterozygous Mutations in NFKB1 Lead to Diverse Immunologic Phenotypes. J Allergy Clin Immunol (2017) 140:782-96. doi: 10.1016/ j.jaci.2016.10.054

16. Fliegauf M, Krüger R, Steiner S, Hanitsch LG, Büchel S, Wahn V, et al. Grimbacher B. A Pathogenic Missense Variant in NFKB1 Causes Common Variable Immunodeficiency Due to Detrimental Protein Damage. Front Immunol (2021) 12:621503. doi: 10.3389/fimmu.2021.621503

17. Li J, Lei W-T, Zhang P, Rapaport F, Seeleuthner Y, Lyu B, et al. Biochemically Deleterious Human NFKB1 Variants Underlie an Autosomal Dominant Form of Common Variable Immunodeficiency. J Exp Med (2021) 218:e20210566. doi: $10.1084 /$ jem.20210566

18. Tuijnenburg P, Lango Allen H, Burns SO, Greene D, Jansen MH, Staples E, et al. Loss-Of-Function Nuclear Factor $\kappa b$ Subunit 1 (NFKB1) Variants Are the Most Common Monogenic Cause of Common Variable Immunodeficiency in Europeans. J Allergy Clin Immunol (2018) 142:1285-96. doi: 10.1016/j.jaci. 2018.01.039

19. Durandy A, Kracker S, Fischer A. Primary Antibody Deficiencies. Nat Rev Immunol (2013) 13:519-33. doi: 10.1038/nri3466

20. Tuijnenburg P, Lango Allen H, Burns SO, Greene D, Jansen MH, Staples E et al. Loss-Of-Function Nuclear Factor $\mathrm{kb}$ Subunit 1 (NFKB1) Variants Are the Most Common Monogenic Cause of Common Variable Immunodeficiency in Europeans. J Allergy Clin Immunol (2018) 142:6749. doi: 10.1016/ j.jaci.2018.01.039

21. Miraghazadeh B, Cook MC. Nuclear Factor-kappaB in Autoimmunity: Man and Mouse. Front Immunol (2018) 9:613. doi: 10.3389/fimmu.2018.00613

22. Snapper CM, Zelazowski P, Rosas FR, Kehry MR, Tian M, Baltimore D, et al. B Cells From P50/NF-кb Knockout Mice Have Selective Defects in 
Proliferation, Differentiation, Germ-Line CH Transcription, and Ig Class Switching. J Immunol (1996) 156.

Conflict of Interest: The authors declare that the research was conducted in the absence of any commercial or financial relationships that could be construed as a potential conflict of interest.

Publisher's Note: All claims expressed in this article are solely those of the authors and do not necessarily represent those of their affiliated organizations, or those of the publisher, the editors and the reviewers. Any product that may be evaluated in this article, or claim that may be made by its manufacturer, is not guaranteed or endorsed by the publisher.

Copyright (c) 2021 Anim, Sogkas, Schmidt, Dubrowinskaja, Witte, Schmidt and Atschekzei. This is an open-access article distributed under the terms of the Creative Commons Attribution License (CC BY). The use, distribution or reproduction in other forums is permitted, provided the original author(s) and the copyright owner(s) are credited and that the original publication in this journal is cited, in accordance with accepted academic practice. No use, distribution or reproduction is permitted which does not comply with these terms. 